\title{
BREAST CANCER SEGMENTATION OF MAMMOGRAPHICS IMAGES USING GENERATIVE ADVERSARIAL NETWORK
}

\author{
$\underline{\text { Swathi }}^{1}$ and T. Christy Bobby ${ }^{2}$ \\ ${ }^{1}$ Department of Computer Science and Engineering, \\ ${ }^{2}$ Department of Electronics and Communication Engineering, \\ M.S. Ramaiah University of Applied Sciences, Bengaluru, Karnataka, India.
}

Corresponding Author:swathi02murthy@gmail.com
Email:swathi02murthy@gmail.com
https://doi.org/10.34107/BiomedSciInstrum.57.04247

\begin{abstract}
Segmentation of breast cancer tumor plays an important role in identifying the location of the tumor, to know the shape of tumor and hence the stage of breast cancer. This paper deals with the segmentation of tumor from whole mammographic mass images using Generative Adversarial Network (GAN). A mini dataset was considered with mammograms and their corresponding ground truth images. Pre-processing like image format conversion, enhancement, pectoral muscle removal and resizing was performed on raw mammogram images. GANs have two neural nets called generative and discriminative networks that compete against each other to obtain the segmentation output. PIX2PIX is a conditional GAN variant which has U-Net as the Generator network and a simple deep neural net as the discriminator. The input to the network was pair of preprocessed mass image and the associated ground truth. A binary image with highlighted tumor was obtained as output. The performance of GAN was evaluated by plotting Generator and discriminator loss. The segmented output was compared with corresponding ground truth. Metrics like Jaccard index, Jaccard distance and Dice-coefficient were calculated. A Dicecoefficient and Jaccard index of $90 \%$ and $88.38 \%$ was achieved. In future, higher accuracy could be achieved by involving larger dataset to make the system robust.
\end{abstract}

Keywords: Generative Adversarial Network, Machine learning, Mammograms, Pix2Pix, Segmentation, U-Net

\section{INTRODUCTION}

Breast cancer is a group of diseases in which cells in breast tissue change and divide uncontrolled, typically resulting in a lump or mass. Breast cancer is the most common type of cancer in women. Diagnosis of breast cancer will be of great help to save millions of lives by predicting cancer survival rates [1]. Accurate segmentation is very necessary for classification of tumor shape and identification of stage of the cancer. Early detection and diagnosis is the best and most effective strategy to control the tumor progression. It is important to assist radiologist in the mammographic lesion interpretation. The shape of the tumor such as lobular, oval, irregular, and round can be known if the tumor is segmented accurately [2]. It also assists in surgeries, specialist driven procedures and therapies such as chemotherapy, radiation therapy and stem cell transplants. Prescriptive treatments include targeted therapy, hormonal therapy and immunotherapy. Tumor segmentation would greatly help in targeted therapy which is specific to cancer cells [3]. Mammogram is the basic and most widely used method for breast cancer screening. A mammogram is an X-ray image of the breast that can detect breast cancer up to 2 years before it can be felt by physical examination. Screening mammogram looks at the whole breast while a diagnostic mammogram examines one section in greater detail, usually by getting more images of a small area to visualize it better [4]. A mammogram is judged based on the Breast Imaging Reporting and Data System number [BIRADS] which is a number used to indicate the radiologist's impression of a mammogram. 